\title{
Coparticipando en el conocimiento: la ampliación de la inteligencia humana con las tecnologías inteligentes
}

\section{Gavriel Salomon, David N. Perkins y Tamar Globerson}

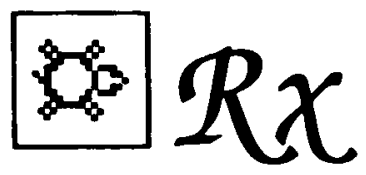

Utilizar un ordenador supone una simbiosis de nuestra inteligencia con una berramienta externa sin la cual la mente contaría sólo con sus propios medios y no funcionaría igual.

Por otra parte, algunos de los procedimientos de uso del ordenador pasan de becho a interiorizarse, a incorporarse autónomamente a la mente.

Este artículo, que permite analizar ambos bechos, nos ayuda a tener claro el papel del ordenador en el aprendizaje $y$ funcionamiento cognitivo

Las personas se han dedicado a producir máquinas cada vez más "inteligentes" ¿Es posible que estas máquinas puedan a su vez hacer más inteligentes a las personas? O con más precisión, dado el uso cada vez más extendido en la educación, de programas de ordenadores inteligentes, de máquinas y de tecnologías relacionadas, puede que sea el momento oportuno para preguntar si estos instrumentos influyen en el rendimiento intelectual y en la capacidad de los estudiantes. Además, algunos educadores han visto en las tecnologías inteligentes la primera visión de un nuevo tipo de educación capaz de facultar y liberar la mente como no lo ha hecho modelo alguno de práctica educativa hasta ahora (Papert, 1980, 1987; Pea, 1987). En vista del auge de tales ambiciones educativas, la cuestión del impacto de las tecnologías inteligentes en el razonamiento y en el aprendizaje humanos adquiere mayor interés cuando existe la posibilidad inminente de obtener respuestas empíricas.

Pero ante todo debemos aclarar dos puntos. Primero, al decir «tecnologías inteligentes» no nos referimos necesariamente a «inteligencia artificial». Hay muchas tecnologías que no se considerarían ejemplos de inteligencia artificial, pero que en el sentido nuestro son tecnologías inteligentes. Por ejemplo, la calculadora manual corriente. No tiene nada de inteligencia artificial, pero realiza 
una operación cognitiva por cuenta del usuario y pudiendo ser considerada así como socio de éste en lo que Pea (1989) ha designado como «inteligencia distribuida». En segundo lugar, aunque el foco de nuestra atención sea la tecnología de los ordenadores, nos damos cuenta que esta tecnología EN SÍ carece de interés. Lo que sí es interesante, y puede afectar el intelecto de los estudiantes, es el tipo de programa y de ordenador que puede emplearse con esta tecnología, así como el tipo de actividad que puede ofrecer. Así, aunque hablemos de «tecnología de los ordenadores», lo hacemos únicamente en aras de la brevedad pero, como se verá, nuestra atención se centra sobre todo en los instrumentos utilizados con los ordenadores, en los programas, y en las actividades que éstos hacen posibles.

Teniendo en cuenta estos puntos, giramos nuestro interés hacia la influencia de las tecnologías inteligentes en el rendimiento intelectual y la capacidad de las personas. Nos proponemos esbozar un sistema conceptual que incluye varias distinciones fundamentales. Distinguimos en primer lugar, entre dos tipos de efectos cognitivos: los efectos que se obtienen EN CONJUNCION CON la tecnología en el curso de la colaboración intelectual con ella, y los efectos PROCEDENTES DE la tecnología, en términos del residuo cognitivo transferible dejado por la colaboración, tras la forma de un mayor dominio de habilidades y estrategias. Observamos que los efectos cognitivos logrados con los ordenadores dependen en gran medida del grado de implicación de los estudiantes en las tareas proporcionadas por estas máquinas, y que existe la posibilidad de mejorar cualitativamente el rendimiento del conjunto aprendiz-tecnología. Esto pone a prueba nuestros conceptos de la capacidad. ¿Es una medida del talento individual (un concepto analítico)? ¿O es más bien una medida del rendimiento cuando se disponga de la tecnología adecuada (un concepto sistemático)? Si tenemos en cuenta que el uso de la voluntad y de la razón en la vida real constituye $-y$ va a constituir- un asunto que concierne principalmente al individuo, desarrollamos entonces el concepto analítico de la capacidad, a través del debate de cómo se pueden conseguir los efectos cognitivos transferibles de la tecnología, aportando pruebas de distintas procedencias. Observamos que es preciso replantear nuestra pregunta inicial acerca de las tecnologías y la mente. En lugar de preguntar cómo la tecnología afecta la mente de forma «natural», en la forma en que los sabios estudiaban los efectos cognitivos del saber leer y escribir, debería preguntarse cómo puede conseguirse que la asociación estudiante-ordenador pueda dar lugar a residuos cognitivos transferibles. Pero cualquier intento de contestar a una pregunta de esta índole implica una ampliación del campo de la investigación. No hay tecnología alguna de ordenador que por sí misma afecte la manera de pensar. Debemos tomar en cuenta, teórica y prácticamente, la totalidad del entorno social y cultural de la enseñanza. Sugerimos que el perfil conceptual que presentamos aquí podría facilitar el diseño de la investigación educacional y el desarrollo de nuevas teorías en este campo.

\section{LOS EFECTOS DE LA TECNOLOGIA INTELIGENTE: DOS ASPECTOS CONTRASTADOS}

Debido al auge de las Tecnologías inteligentes, inmediatamente surgen interrogantes sobre el impacto que éstos puedan tener en los modos de pensar y de aprender. Para que la pregunta tenga sentido, hay que distinguir entre dos 
formas muy distintas en que las tecnologías inteligentes podrían afectar la capacidad del intelecto humano. Una de las formas tiene que ver con los cambios en el rendimiento, manifestados por los estudiantes EN EL CURSO DE su actividad asistida por un programa o un ordenador - por ejemplo, el grado de sofisticación de los hipótesis que ellos generan en el trabajo con un ordenador constructor de modelos (Mandinach, 1989) -; En este, como en otros muchos casos, el hecho de trabajar con una máquina inteligente influye EN LO QUE HACEN los estudiantes, en la CALIDAD de lo que hacen, y en CUANDO lo hacen (Pea, 1985). Vamos a llamar «EFECTOS CON LA TECNOLOGIA» a estos posibles resultados. Otro significado del término «efecto» se refiere a las transformaciones relativamente duraderas que se observan en las capacidades cognitivas generales de los estudiantes COMO CONSECUENCIA de su interacción con una tecnología inteligente. A este tipo de efectos pertenecen los cambios posteriores, en el dominio del conocimiento, de la habilidad, o bien de la profundidad de la comprensión, después de que el estudiante se aleje del ordenador. Vamos a llamar a estos resultados «Efectos DE LA TECNOLOGIA».

La diferencia entre los dos tipos de efectos se ve reflejado en el caso de un experto operador de un ábaco. Por un lado, la persona demuestra una mayor habilidad aritmética mientras trabaja con el ábaco - (el efecto CON el ábaco) - - Luego es posible que demuestre una mayor capacidad matemática cuando hace sus cálculos sin el ábaco, una consecuencia de la interiorización de los procedimientos facilitados inicialmente por el ábaco - (el efecto DE la herramienta)- Otro ejemplo podría ser el de un procesador de textos muy inteligente. Por un lado, los estudiantes podrían escribir mejor utilizando el ordenador; por otro lado, un ordenador tan inteligente podría enseñar principios básicos acerca del arte de escribir que ellos podrían aplicar ampliamente cuando les tocara escribir con una máquina muy simple. Esto sugiere de nuevo el efecto obtenido DE la máquina.

La diferencia entre estos dos tipos de efectos encuentra un paralelo en el contraste entre las opiniones de expertos que han estudiado el impacto de la alfabetización en el conocimiento. Havelock (1982) investigó los efectos CON la alfabetización, preguntando cómo el saber leer y escribir ha superado una nueva definición de los roles y de las funciones de la memoria, y cómo en consecuencia la memoria se ha visto reforzada. Pero no consideró cómo el saber leer y escribir afecta la capacidad de la memoria. Por otra parte, Scribner y Cole (1931) - para mencionar sólo uno de los estudios importantes- estudiaron los efectos del saber leer y escribir sobre otras funciones y habilidades mentales, valorando así las consecuencias cognitivas del saber leer y escribir.

Aunque en algunas fuentes examinan el impacto de las tecnologías precedentes y se enfrentan con la pregunta de el CON y algunos con el DE. En general no se han confrontado estos dos aspectos para hacer una comparación sistemática. Es importante, sin embargo, no descuidar el uno a favor del otro, y no confundir los dos, como se ha hecho a veces. Según las circunstancias, uno u otro aspecto podría ser enormemente más importante. Nos podemos encontrar que el lápiz, la regla de cálculo y el procesador de textos supongan una definición totalmente nueva de las tareas de memorización, cálculo, escritura, hasta, quizás, un aumento del rendimiento de manera absoluta. Pero esto no significa inevitablemente que estos artilugios dejen un residuo cognitivo en forma de una mayor capacidad de recordar información, calcular o escribir en su ausencia. Actualmente es posible planificar, diseñar, escribir, experimentar y simular de 
maneras que antes ni se imaginaba. Pero ¿esta asociación significa que los estudiantes sean más inteligentes, mejores comunicadores, y con mejor (o quizás peor) disposición para aprender?

Vamos a examinar estas cuestiones, empezando con una discusión de los efectos cognitivos obtenidos CON la tecnología, seguida de una discusión de los efectos DE la tecnología, y terminando con un breve examen de los contextos más amplios dentro de los cuales conviene estudiar dichos efectos.

\section{EFECTOS OBTENIDOS CON LA TECNOLOGIA: COLABORACION INTELECTUAL}

Las tecnologías pueden dividirse aproximadamente en dos grupos, según el uso que se hace de ellas: hay máquinas que trabajan para nosotros, y hay herramientas con las que nosotros trabajamos (Ellul, 1964). El motor - (no el coche en su totalidad) - el reloj y el piloto automático trabajan para nosotros. El lápiz, el azadón, el microscopio, la cámara fotográfica, la regla de cálculo, el procesador y el paquete estadístico computerizado solicitan que trabajemos con ellos; sin nuestra participación son de poca utilidad. Este último tipo de tecnología nos interesa mucho aquí, puesto que nos proporciona una asocación intelectual en la que los resultados dependen mucho del esfuerzo del conjunto.

La colaboración con los ordenadores supone la existencia de los tres componentes de la asociación humana: (a) una división complementaria del trabajo que (b) se hace interdependiente, y que (c) se desarrolla en el tiempo. La asociación es, además, verdaderamente intelectual. Según la definición del concepto de tecnología inteligente, la herramienta asume una parte de la carga intelectual del tratamiento de la información. Por ejemplo, las hojas de expansión, los paquetes estadísticos y los equipos para gráficos ponen al alcance del experto unas facilidades de gran valor que ahorran el esfuerzo cognitivo necesario para conseguir un resultado profesional; hasta un novato con poca experiencia puede elaborar un producto respetable.

Además, un inexperto podría beneficiarse del uso de ciertos programas de ordenador que apoyan los procesos cognitivos, ya que las operaciones de razonamiento avanzado requieren una automatización de las de orden inferior (Anderson, 1983). La colaboración con el ordenador podría efectuar algunas de estas operaciones menores, con lo que se evitaría la necesidad de llegar primero a una automatización. Los principiantes quedarían libres para abordar actividades cognitivas que estarían fuera de su alcance sin la colaboración tecnológica (Olson, 1988). Ampliando la cuestión del potencial de los ordenadores que apoyan el aprendizaje de los principiantes. Un programa como STELLA, por ejemplo, (Richmond, 1985), permite la construcción de modelos de bases matemáticas para sistemas económicos, históricos, ecológicos o de transporte. Las variables, los valores y sus relaciones son introducidos en el modelo por los alumnos. Los estudiantes no necesitan memorizar nada - un alivio en vista de la complejidad de los modelos que pueden ser elaborados con el programa- pero ellos pueden genera y probar las hipótesis más inverosímiles acerca de las interrelaciones de los datos conceptuales (Mandinach, 1989). El estudio de las asignaturas deja de ser una memorización de datos inconexos, que se hace totalmente innecesaria, y pasa a la búsqueda de maneras de interrelacionarlos. El sistema permite, además, que los estudiantes organicen las ideas en profundidad en vez de tra- 
tarlas superficialmetne, una capacidad que es más característica del experto que del principiante (Chi, Feltovitch y Glaser, 1981).

Basándonos en estos ejemplos, podemos llamar a los ordenadores que ofrecen una asociación o colaboración intelectual «Instrumentos cognitivos» (Pea, 1985) o «Tecnologías de la mente». Potencialmente, permiten al estudiante funcionar a un nivel que trasciende las limitaciones de su sistema cognitivo. Contando con estos instrumentos, el aprendiz puede meterse en la comprobación de hipótesis, el diseño de ambientes ecológicos hipotéticos, ensayos de laboratorio, planificación, escritura inteligentemente orientada, la resolución de problemas matemáticos, y la construcción de modelos, a niveles que hasta ahora eran poco accesibles. En realidad, podemos argumentar que trabajar con el ordenador adecuado sirve para mucho más que permitir al principiante hacer la misma cosa pero con más rapidez y menos esfuerzo. La existencia de tales sistemas podrían significar una nueva definición y una nueva estructuración de la tarea de aprender o de operar, de la misma manera que el lápiz ha significado una restructuración cualitativa del acto de memorizar (Cole y Griffin, 1980). En suma, la colaboración intelectual con estos ordenadores puede cambiar la relación entre la adquisición de conocimientos preparados y la construcción de nuevos conocimientos, a favor de este último (Pea, 1987). La obra de una persona en colaboración con la tecnología podría ser mucho más «inteligente» que la obra de la persona a solas.

\section{LA COLABORACION CONSCIENTE}

Debemos advertir que hemos puesto el énfasis en los efectos que PODRIAN lograrse con la asociación persona/ordenador. Si observamos las actividades de los estudiantes con programas como el Learning Tool (Kozma y Van Roekel, 1986) - STELLA, resulta evidente la posibilidad de una colaboración inteligente. Otra custión sería la frecuencia con que se aprovechan tales oportunidades. No se puede suponer que se aprovechen de manera automática, ni siquiera cuando existen las condiciones idóneas (Perkins, 1985). Desgraciadamente, cualquier colaboración exige esfuerzo, y la colaboración intelectual entre personas y teconología no es una excepción.

Aceptamos como principio básico que si la colaboración ha de conseguir niveles de rendimiento superiores, los procesos mentales de la persona deben ser de tipo no-automático (Shriffen y Schneider, 1977). Estos procesos están controlados por la voluntad del alumno, no por la tarea ni tampoco por los materiales (Schneider y Fisk, 1984), y además, exigen un esfuerzo. El empleo de los procesos, no-automáticos y difíciles, y en consecuencia dirigidos metacognitivamente, ha sido definido como «mindfulness» - atención voluntaria (Salomon y Globerson, 1987) - Contrasta con un estado de poca atención, caracterizado por una dependencia ciega de los aspectos marcadamente estructurales de una situación, sin fijarse en sus características singulares y novedosas (Langer, 1989).

Esta atención es parecida al «empleo de una alta capacidad cognitiva» (Britton, Glynn, Meyer y Penland, 1982) y al «esfuerzo constructivo en el aprendizaje» (Bereiter y Tinker, 1988), que son estados en los que la persona no depende de procesos ya automatizados sino de procesos controlados por la determinación de la tarea. Por ejemplo, cuando los estudiantes en su trabajo con el Lear- 
ning Tool piensan seriamente en métodos novedosos de relacionar distintos conceptos entre sí, considerando los atributos de estos conceptos, están utilizando el programa con atención; pero si se limitan a desplegar los conceptos como si se tratara de un mapa, y luego hacen una interrelación al azar, esto sería un empleo del programa sin la debida atención.

Según varios investigadores — (por ej. Bereiter y Tinker, 1988; Langer, 1989; Salomon y Leigh, 1984)-, la implicación atenta y voluntaria en una tarea obliga a los estudiantes a agilizar su inteligencia, generar mayor número de deducciones originales, y memorizar más y mejor el material encontrado. Aunque parezca que esto sea aplicable de modo general a cualquier aprendizaje y a cualquier trabajo con materiales nuevos, evidentemente tiene menos importancia cuando los estudiantes trabajan bajo supervisión, y más importancia cuando trabajan solos. En la primera situación, los procesos mentales están canalizados por procesos didácticos, mientras que en la segunda, el uso de procesos noautomáticos depende en gran medida de la propia iniciativa del estudiante.

La idea misma de trabajar con un ordenador se basa en la suposición de que los usuarios exploren, diseñen, investiguen, escriban o comprueben hipótesis con métodos que acoplan la inteligencia de la máquina con la suya, en una participación consciente en la tarea. La consecuencia es que el mayor rendimiento en el trabajo con un ordenador sólo se logra cuando los estudiantes operan con atención y a conciencia.

Este estado de atención o concentración en la colaboración con un ordenador proviene como mínimo de dos corrientes. Una de estas corrientes es la tendencia general de las personas de ser procesadores atentos de la información que reciben (Cacioppo y Petty, 1982). Otra corriente, que en nuestro estudio es de mayor importancia, es la combinación de materiales, tarea y medios que estimulan la atención. Por ejemplo, Malone y Lepper (1987) estudiaron las cualidades de los juegos de ordenador que aumentan la motivación intrínseca, esperando que los mismos aumentaran también la complicidad en el proceso. Algunas de estas cualidades - control de la actividad, interactividad, resultados inmediatos, objetivos escalonados, conflicto y una moderada incertidumbre-, se encuentran no sólo en los juegos sino también en los ordenadores inteligentes. De hecho, esto es una consecuencia de su naturaleza: presentan al usuario unos puntos de elección que invitan a la reflexión, y al aprendiz unos puntos de conflicto destinados a provocar una experimentación mental. (Gelman y Brown, 1986). Sin embargo, esto no significa que el programa se pueda usar como herramienta en el tanteo; cuanto más abiertas sean las actividades proporcionadas por el ordenador, tanto más libre se encuentra el estudiante para entrar -o no entrar - en complicidad intencionada con ellas.

En su tesis doctoral en la universidad de Tel-Aviv en 1988, Rachel Mintz apoyó este último argumento. Ella encontró importantes correlaciones entre el esfuerzo (según los interesados) y los resultados cuando los estudiantes trabajaban con un simulador ecológico plenamente interactivo. Cuando trabajaban con un simulador no-interactivo, fue la capacidad inicial, no el gasto de esfuerzo, lo que mejor pronosticaba el efecto educativo. Estas observaciones hacen pensar que algunos estudiantes, posiblemente aquellos que prestan poca atención, (Cacioppo y Petty, 1982; Salomon y Globerson, 1982), no se esfuerzan, ni siquiera cuando tienen la posibilidad de una verdadera complicidad con un ordenador que comprometería, sin embargo, a otros estudiantes en un proceso de exploración atenta. En palabras de Chanovith y Langer (1980): «El :mbiente 
aparentemente estructurado SUGIERE ciertos modos de participación intelectual, pero no DICTA ese modo». (pág. 102).

En resumen, los ordenadores inteligentes ofrecen una posibilidad de colaboración capaz de ampliar el rendimiento intelectual del usuario. Pero el grado de realización o cumplimiento de este potencial depende en gran medida del compromiso voluntario del usuario. No depende solamente de la «pareja» de interacción estudiante/ordenador sino de COMO se emprende la colaboración.

\section{LA CUESTION DE LA CAPACIDAD}

Del comproniso voluntario de asociación entre el usuario y el ordenador, se puede esperar un resultado notable. Pero dicha asociación pone en tela de juicio las teorías tradicionales acerca de la capacidad. Cualquiera que sea la definición empleada, se piensa normalmente que es el potencial de la mente de la persona, la propiedad del individuo. Pero una vez efectuada la asociación entre las tecnologías inteligentes y la capacidad del individuo, el énfasis podría desplazarse hacia un examen del rendimiento del conjunto. Al fin y al cabo, es el SISTEMA, no el individuo a solas, que ejecuta la tarea intelectual (Pea, 1987, 1989).

Este nuevo concepto de la capacidad humana parece a primera vista bastante original. Sin embargo, un examen más detallado demuestra que, al menos implícitamente, la hemos aceptado siempre. Como lo indica Olson (1986): «Casi todas las formas de cognición humana exigen el uso productivo e imaginativo de alguna tecnología. El tratar de caracterizar la inteligencia independientemente de esas tecnologías parece un error fundamental» (pág. 356). Por ejemplo, no se nos ocurriría juzgar la capacidad artística de una persona sin tomar en consideración algún medio como pinceles y pintura. Como dijo Pea (1989), con el empleo de los ordenadores apropiados, queda distribuida la capacidad mediante una «descarga» de algunas de las operaciones mentales sobre el ambiente artifactual.

Entonces tendríamos que aceptar, por analogía, el concepto de capacidad intelectual como una propiedad de un conjunto - una persona trabajando en colaboración con una tecnología inteligente- Sin embargo, esta extrapolación no es tan simple como puede parecer a primera vista. ¿Qué diríamos de una asociación entre una persona y una tecnología inteligente que produce resultados, pero que deja a la persona con una idea simplista y errónea cuando tiene que trabajar sin la tecnología (por ej. Gentner y Gentner, 1983)? Además, ¿qué ocurre si el componente tecnológico posee la pericia suficiente para REDUCIR la contribución intelectual del socio humano, como es el caso hoy día con los sistemas de pilotaje que reemplazan al piloto humano en un aterrizaje peligroso? ¿Qué ocurriría si los sistemas expertos de diagnosis llegaran a ser tan perfectos que el médico novato no fuera más que un manipulador de datos?

Estos dilemas pueden ser resueltos si se consideran dos maneras de evaluar la inteligencia de colaboración entre la persona y el ordenador: la manera SIS. TEMATICA y ANALITICA. El enfoque sistemático estudia el rendimiento del sistema en su conjunto, juzgando los productos de la persona de la de la tecnología. Al contrario, la manera analítica estudia los tipos específicos del proceso mental que constituye la contribución del ser humano. Por ejemplo, ¿cómo procede un principiante, equipado de un simulador que permite manipular paque- 
tes complejos de variables, para comprobar las hipótesis acerca de la interrelación de variables ecológicas (por ej. Mints, 1988)?

Desde el punto de vista sistemático, lo que vale es el nivel general del rendimiento del sistema, no el del individuo que participa; la capacidad en este caso es el producto creado por el conjunto persona-máquina. El punto ciego de este enfoque es que cuando los ordenadores llegan a ser lo suficientemente inteligentes, la contribución de la persona se pierde de vista, y pueden presentarse los casos inquietantes a los que nos hemos referido.

Con el enfoque analítico, por el contrario, podemos continuar con la concepción de la capacidad como una propiedad de las personas, y podemos valorar la capacidad manifestada por ellas en su trabajo con el ordenador. Como afirma Vygotsky (1978), la posibilidad de trabajar con un ordenador inteligente podría considerarse como una invitación para operar dentro de una zona de desarrollo próximo. La colaboración con la tecnología es parecida al trabajo con un colega mejor dotado; permite a los estudiantes atentos tomar parte en proce- sos cognitivos que superan a los que ellos podrían conseguir sin dicha colaboración. Se valora todavía el rendimiento del individuo, pero en condiciones que le permiten estirar sus músculos cognitivos al máximo. La cuestión de cómo definir la capacidad puede, por lo tanto, recibir dos respuestas. Una adopta el enfoque sistemático valorando el producto, el rendimiento, de las capacidades unidas, de la persona con elordenador. La otra emplea el enfoque analítico, valorando los tipos de actividad mental aportados por el individuo en colaboración con el ordenador inteligente. Este último enfoque se orienta más hacia el estudio del potencial humano y de factores de interés educativo. Favorece el empleo educacional de los programas cognitivos que permiten una actividad mental de orden superior, como podría ser el caso de STELLA y el Learning Tool, más que de aquellos programas que mejoran el rendimiento del sistema pero no el del estudiante.

\section{EFECTOS DE LA TECNOLOGIA: LOGRAR UN RESIDUO COGNITIVO}

La distinción entre las perspectivas sistemáticas y analíticas ayuda a resolver el problema de definición de capacidad cuando las personas trabajan CON las tecnologías, pero no explica los efectos DE las tecnologías sobre la cognición. Este último es un asunto totalmente distinto. Un sistema diseñado para mejorar una diagnosis o hacer una nueva definición del proceso de la escritura cuando se trabaja CON dicho sistema no es necesariamente el mejor método para cultivar la habilidad del individuo en diagnosticar o en escribir. Se podría argumentar, en este punto, que la diferencia entre los efectos obtenidos CON el programa y los obtenidos COMO CONSECUENCIA del mismo no es más que temporal. Al fin y al cabo, según este argumento, los ordenadores del futuro serán tan eficaces que todos los efectos procederán de su uso, y en consecuencia, la cuestión del residuo cognitivo dejado por la colaboración será más bien irrelevante.

En realidad, el contraste entre los dos enfoques crea un dilema de elección. Quizás deberíamos limitarnos a apuntar a los efectos obtenidos CON una tecnología por cuyo medio se obtiene una verdadera distribución de la inteligencia (Pea, 1989), poniendo el énfasis de esta forma en el aspecto sistemático de la capacidad humana. Si las personas-más-la-tecnología pueden efectuar tareas que 
previamente les resultaban imposibles, entonces la cuestión de los residuos dejados por la colaboración con la tecnología podría carecer de importancia. Olson, Torrance y Hildyard (1985) argumentaron con respecto al saber leer y escribir: «Es engañoso pensar en el saber leer y escribir en términos de sus consecuencias. Lo que importa es lo que hacen las personas con este saber, no el efecto que obra sobre ellas el saber» (pág. 15); y lo mismo se podría afirmar en lo referente a los ordenadores.

Sin embargo, esto parece demasiado radical en el estado actual de la tecnología. Mientras las tecnologías inteligentes no sean tan generalizadas como el lápiz y el papel -lo que está todavía muy lejos- es preciso examinar la actuación de una persona alejada de las tecnologías inteligentes. $\mathrm{E}$ incluso si éstas llegaran a ser tan corrientes como el lápiz, los estudiantes tendrían que solucionar todavía un sinfín de problemas, construir mentalmente nuevos tipos de conocimiento y tomar decisiones, para todo lo cual no dispondrían de tecnología. Ante preguntas como «Qué más debería preparar para el examen?», «¿Qué van a pensar mis lectores de este argumento?», "Cómo hago concordar mis ideas actuales sobre la URSS con las que tenía anteriormente?», o «Cómo es que la electricidad no se agota subiendo por el Empire State Building?», hace falta una mente independiente y capaz de pensar, no una mente que depende continuamente de una asociación con la tecnología, por muy inteligente que sea.

Los efectos cognitivos DE la interacción con programas de ordenador pertenecen al cultivo de tales habilidades y capacidades. Consideremos la posibilidad de que la colaboración intelectual con un ordenador deje un residuo cognitivo TRANSFERIBLE, por ejemplo en forma de una capacidad generalizada de autorregulación y orientación (Bereiter y Scardamalia, 1987; Salomon, Globerson y Guterman, 1990). Una mejoría de esta índole serviría al individuo en una multitud de situaciones, sobre todo cuando se encontrara solo. De modo parecido, el mayor dominio de estrategias y de habilidades podría permitirle acceder a actividades de orden superior en colaboraciones posteriores con máquinas inteligentes.

En consecuencia, el impacto DE una tecnología es tan importante como el rendimiento logrado CON ella. Naturalmente queremos ver a los estudiantes trabajar mejor CON los programas de ordenador, pero queremos ver también un impacto cognitivo positivo $\mathrm{DE}$ estos programas. Los estudiantes deberían llegar a ser mejores escritores, no sólo cuando están trabajando con un programa de escritura o de desarrollo de ideas; también deberían llegar a escribir mejor con un simple procesador de textos que no ofrece ninguna orientación inteligente, o incluso con lápiz y papel. Para relacionar esta preocupación por la concepción de la inteligencia, vista desde el ángulo educacional, tenemos que adoptar el enfoque analítico, destacando los residuos cognitivos de la colaboración intelectual, aquellos que los estudiantes retienen tras la experiencia con la tecnología.

La posibilidad de un residuo cognitivo descansa, sin embargo, en un supuesto importante: que las capacidades de razonamiento de un ordenador superior - sean las actividades en el curso del trabajo con un ordenador inteligente $o$ las explícitamente modeladas por el mismo- puedan desarrollarse y luego ser transferidas a otras situaciones, quizás diferentes o en algún caso similares. Esta expectativa de un residuo cognitivo transferible descansa a su vez en otro supuesto más básico, de que, en contra de algunas opiniones (por ej., J.S. Brown, Collins y Duguid, 1989), las habilidades cognitivas del tipo que uno quisiera 
cultivar en la escuela no han de estar necesariamente ligadas a un contexto o «situadas» (Perkins y Salomon, 1989). La cuestión está en si existen pruebas de que el uso de la tecnología produzca tales efectos secundarios.

\section{TECNOLOGIAS INTELIGENTES Y LA CUESTION DE TRANSFERENCIA}

En el caso de las tecnologías «antiguas», no es fácil llegar a conclusiones. Tales tecnologías - (escritura, TV, etc.) - son tan extendidas, y su presencia tiene tanta correlación con otros factores sociológicos, que resulta difícil organizar manipulaciones experimentales, y es difícil también encontrar «experimentos naturales» que sean razonablemente claros. Una rara excepción es un estudio reciente, amplio y casi experimental, de los efectos del saber leer y escribir sobre el desarrollo de procesos cognitivos (Scribner y Cole, 1981). Una tribu de Liberia, los Vai, tienen un sistema de escritura conocido entre muchos miembros de la cultura; pero no tienen sistema alguno de educación formal. Esta situación ofrece la posibilidad de separar el saber leer y escribir de la variable compleja de la escolaridad. Los resultados del estudio de Scribner y Cole fueron provocativos: no se encontró consecuencia cognitiva alguna del saber leer y escribir.

Pero, como indican los autores, el leer y escribir no juega un papel social y cultural tan central entre los Vai como es el caso en nuestra cultura. Los autores sugieren que la influencia de la tecnología sobre la mente sólo viene dada cuando la tecnología es de importancia vital, y cuando sirve para muchas finalidades en la vida de la comunidad. Además, se observaron efectos secundarios en los MAESTROS informales de la escritura Vai. Puede ser que los aprendices normales no dedicaran la atención suficiente para que el impacto cognitivo fuera más amplio; sus maestros, en cambio, peleando con las demandas de la enseñanza, sí que ponían atención.

Incluso cuando la tecnología juega un papel social central, es posible que no afecte inmediatamente a las mentes. Es posible que el saber leer y escribir nos haya obligado a distinguir claramente entre lo que se dice o se escribe y lo que se quiere decir con ello (por ej., Olson, 1986). En las sociedades iletradas, según este autor, no se hace esta distinción. Pero tales efectos, suponiendo que sean el resultado de la alfabetización y la prensa, no se produjeron de la noche a la mañana, ni tampoco durante el tiempo en que una generación empezaba a leer y escribir. Desgraciadamente, los procesos largos no se prestan a la observación ni a sistemas de medida. Los análisis socio-históricos, como los de Havelock (1982) o de Goody (1977), aunque muy sugerentes, no ofrecen la evidencia empírica de los efectos de la tecnología sobre la mente que uno quisiera encontrar.

Es un hecho que los efectos de la tecnología son difíciles de valorar en el caso de las tecnologías antiguas. Las nuevas tecnologías inteligentes, sin embargo, ofrecen mejores perspectivas. En primer lugar es relativamente fácil efectuar pruebas controladas porque la mayoría de las personas han tenido poco contacto con las nuevas tecnologías. Segundo, algunas de las tecnologías inteligentes pretenden cultivar un comportamiento inteligente con una intencionalidad que no existía en las más tradicionales, de escritura o de telecomunicaciones. Algunas tecnologías inteligentes actuales o en fase de desarrollo orientan al usua- 
rio directa y eficazmente en el despliegue de estrategias de razonamiento, de aplicación general, e incluso las cultivan (por ej., Mandinach, 1989; Salomon et al., 1990; Zellermayer, Salomon, Globerson y Givon, en prensa).

Las conclusiones con respecto a lo que definimos como los efectos cogniti. vos DE la tecnología de ordenadores son ambiguos. Mientras algunos estudios demuestran la esperada transferencia de habilidades adquiridas durante la asociación con programas de ordenador (por ej., Levin, Riel, Miyake y Cohen, 1987, Salomon et al, 1990), otros no han encontrado residuo cognitivo alguno (por ej., Pea, Kurland, y Hawkins, 1985), o los han encontrado solamente en estudiantes de alta categoría (por ej., Mandinach y Corno, 1985; ver una crítica por Krendl y Lieberman, 1988). Perkins y Salomon (1987) and Salomon y Perkins, (1989) examinaron algunos estudios en los que habían encontrado residuos cog. nitivos transferibles como resultado de la asociación intelectual con ordenadores y programas u otro tipo de intervenciones didácticos, y otros en los que no habían encontrado tales indicios. Observaron que la diferencia principal entre los dos grupos de estudios está en la manera cualitativa de la participación de los estudiantes en la asociación o la enseñanza. Afirmaron que puede lograrse algún nivel de transferencia tras muchas y diversas prácticas hasta aproximarse a la automaticidad, mediante un proceso que J. Anderson (1983) llamó «generalización de la habilidad». Sin embargo, son pocas las situaciones de enseñanza y de experimentación que llegan a proporcionar este nivel de práctica. Salvo aquellas situaciones en las que los estudiantes se entregan a una abstracción atenta a los procedimientos, a la autorregulación, o a las estrategias que se activan o se modelan en el curso de la colaboración, entonces sí que se produce la transferencia de estas disciplinas. No es frecuente que los usuarios dediquen tanta atención al uso de una tecnología en circunstancias normales, no didácticas; pero la atención se puede provocar. Por ejemplo, en los cursos de programación de ordenadores, donde se ha cultivado por medio del método de instrucción una actitud de abstracción consciente (Salomon y Perkins, 1988), la transferencia es impresionante (Clements y Gullo, 1984). Estas investigaciones con ordenadores (por ej., Salomon et al, 1990) han demostrado más POSIBILIDADES de lograr efectos transferibles de la asociación con ordenadores y programas que las REALIDADES de transferencia en las condiciones normales de la actividad diaria. En la búsqueda de transferencias dentro del ambiente escolar, quizás la pregunta «¿Existe un efecto cognitivo de la tecnología?» no sea la adecuada: la abstracción necesaria no va a producirse espontáneamente.

La pregunta más adecuada podría ser «¿Es posible "fraguar" un efecto cognitivo de la tecnología a través del diseño, la actividad y el entorno, para fomentar esta abstracción consciente de las habilidades y estrategias mentales?».

\section{EL CONTEXTO MAS AMPLIO DE LOS EFECTOS DE LA TECNOLOGIA}

La pregunta inicial $-¿$ Existe algún efecto del ordenador sobre el rendimiento y la capacidad de los estudiantes? - se ha replanteado. Al principio de este trabajo, distinguimos entre los efectos observados $\mathrm{CON}$ los ordenadores y los efectos producidos POR los ordenadores, un contraste que permite enfocar mejor las preguntas, al tiempo que cuestiona nuestras ideas acerca de la capacidad humana. Seguidamente, hemos examinado las condiciones previas para conseguir efectos positivos CON la tecnología y DE la tecnología - la importancia, por ejemplo, de la atención y la abstracción reflexiva. 
Aunque todo esto es relevante, hasta aquí hemos tratado solamente la cuestión de la tecnología en sí y la variable independiente del interés. Hace falta un marco más amplio, ya que el peso de la tecnología en el razonamiento humano depende invevitable y claramente de otros factores que se extienden más allá de la tecnología en sí. Veamos tres contextos, como mínimo, que son relevantes: el normativo, el teórico y el práctico.

\section{EL CONTEXTO NORMATIVO}

Si es posible lograr efectos positivos con la tecnología, ¿no podemos lograr también efectos negativos? March (1987) habla de «incapacitación», como consecuencia del trabajo con herramientas inteligentes que adormecen ciertas habilidades porque el ordenador las hace innecesarias. Algunas de estas habilidades, como por ejemplo, el saber derivar una raíz cuadrada, podrían perderse. $Y$ volviendo a un asunto al que nos referimos anteriormente, ¿qué ocurre si un sistema experto llegase a un tal grado de inteligencia que aniquila la capacidad del médico para diagnosticar?

Es posible que desde el punto de vista sistemático esto no tenga importancia. Lo que importa es la mayor capacidad de diagnóstico del SISTEMA. Pero desde el punto de vista analítico, sobre todo si existen intereses normativos y educacionales, la cuestión requiere un examen cuidadoso. ¿Qué pasaría si el sabio diagnóstico de un médico fuera sustituido por la operación automática de una caja negra? En el afán de producir ordenadores cada vez más inteligentes, podríamos condenar a desaparecer habilidades que preferiríamos conservar. Como ha dicho Rollo May, no todo lo posible es necesariamente deseable. Y en palabras de Sarason (1984): «El hecho de que algo pueda ser estudiado o desarrollado no es suficiente base como para llevarlo a cabo, por muy maravilloso que parezca para la comprensión y el dominio de nuestro mundo (pág. 480).

\section{EL CONTEXTO TEORICO}

Los aspectos normativos señalan una conclusión inevitable: desde el punto de vista de la construcción de teorías, el impacto de las tecnologías inteligentes en las acciones humanas exige teorías de gran envergadura, que tomen en cuenta las variables tecnológicas, psicológicas individuales, y sociales. De hecho, ninguna tecnología -incluida la de los ordenadores - afecta la mente de una persona si no es a través de las operaciones mentales específicas que emplea. Recordando la opinión de Leont'ev, (1981), alumno de Vygotsky, podríamos decir que estas operaciones: proyectar, comprobación de hipótesis, respuestas a las orientaciones metacognitivas inteligentes, se incluyen entre las actividades directivas al logro de los objetivos: programar, escribir, construir modelos... que a su vez son afectados por la cultura que las determina, las aprueba y las promociona. Ni las operaciones mentales, ni las actividades que las aprovechan, existen en un vacío cultural.

Pensemos en la total ausencia de efectos de «la letra» en las cogniciones del pueblo Vai, de Scribner y Cole (1981). Como señalan los autores, el uso del leer y escribir fue bastante limitado si comparáramos con nuestra sociedad. Servía para unas funciones sociales y comerciales de poca importancia; de la letra impresa no dependía nada fundamental, y nada nuevo se había sumado a la cultu- 
ra Vai a través de la escritura. No se puede concluir que el saber leer y escribir no afecte las cogniciones de los practicantes de estos saberes. La conclusión más plausible es que afectan la mente sólo en aquellas culturas que son «letradas» al máximo, donde se practica el leer y escribir en las más variadas circunstancias, en muchísimas actividades y para un sinfín de quehaceres (Scribner y Cole, 1981).

Además, suponemos una interrelación y reciprocidad entre la tecnología, los tipos de actividad, los efectos cognitivos y las funciones sociales. Si uno dispone de una tecnología, lo más probable es que la use. Y con este uso inicial, las actividades anteriores se hacen de otra manera (por ej., escribir con un procesador), se presentan nuevas actividades (por ej., el programar), y se cambian los roles para la mente (por ej., el conocimiento, en vez de ser algo que se posee, se convierte en la acción de recurrir a la base de datos). Es probable que esto, a su vez, afecte el ambiente cultural, lo que llevará a su vez a una nueva definición del papel de la tecnología en la sociedad.

En consecuencia, si los ordenadores llegaran a ser el centro de la educación según predicen algunos como inevitable, toda la cultura de la escuela cambiaría, de un sistema de conocimientos impartidos se podría ir a un régimen de exploración libre y a una re-creación de conocimientos. Desde luego una transformación de esta naturaleza cambiaría a su vez el rango de los ordenadores dentro de la escuela, quedando al descubierto nuevos y sorprendentes roles para ellos. En algunas culturas o instituciones, en ciertos ambientes y subculturas, el ordenador podría llegar a ser la «tecnología definitoria» de la época (Bolter, 1984), que influiría en el tipo de actividades a que la gente se dedicara y la manera de practicarlas. En otros sectores, el ordenador podría quedar como un accesorio poco importante, con lo que sus efectos potenciales serían menores.

El examen de los efectos CON la tecnología inteligente, y los efectos DE esta tecnología, gana interés si se ensancha la perspectiva teórica para incluir el contexto cultural como fuente, no sólo de variables periféricas sino también de aquellas variables que afectan recíprocamente y sumultáneamente unas a otras (Cole, 1985), en lo que Scarr (1985) llamó «una nube de variables correlativas». Es posible que algunos experimentos controlados demuestren la posibilidad de manejar ciertos efectos en condiciones favorables. También estos experimentos podrían sugerir algunos mecanismos que transmitirían los efectos -cierta interiorización, por ejemplo, o una atención provocada-. Sin embargo, tales experimentos no nos indican lo que ocurre - podría ser provocado - en condiciones normales cuando se asumen papeles tan importantes como los contextos culturales, sociales e institucionales.

\section{EL CONTEXTO PRACTICO}

Todo esto lleva a un breve comentario sobre los contextos prácticos en los que la tecnología puede afectar la mente. A la luz de nuestra discusión del contexto teórico, es evidente que sólo se producen efectos mentales profundos provocados por la tecnología inteligente cuando al mismo tiempo se producen cambios notables en la cultura. No se puede esperar impacto importante alguno cuando se practica la misma vieja actividad con una tecnología que hace que se realice esta misma actividad más rápidamente o con mayor facilidad. Es preciso cambiar la actividad, y esto no puede efectuarse en un vacío cultural. 
La tecnología no es el único factor que afecta las mentes; es toda la «nube de variables correlativas» - la tecnología, la actividad, el objetivo, el ambiente, el rol del profesor, la cultura - que ejercen sus efectos combinados. En consecuencia, para manejar un efecto deseable, sea CON una tecnología inteligente - DE la misma, hace falta mucho más que la mera introducción de un nuevo programa u ordenador. Por ejemplo, Daiute $(1985,1988)$ y Daiute y Kruidener (1985) han demostrado que la introducción de procesadores de texto en las aulas produce un efecto muy limitado - principalmente una mayor corrección de la ortografía y gramática-. Con frecuencia los estudiantes encuentran una idea adicional, pero la añaden al final en vez de integrarla en la redacción. En cambio, cuando la tecnología o el profesor ofrece un apunte, una sugerencia directa que invita a una revisión estructural, entonces los estudiantes responden con una nueva versión, más elaborada y de mayor interés.

Otro ejemplo: Papert (1987) describió dos escuelas en donde se introdujo Logo. En una de ellas, se trató el Logo como una asignatura cualquiera; en la otra se utilizó como material más o menos de experimentación. En las dos escuelas se aprendió Logo, pero los tipos de actividades y las culturas del aprendizaje - didáctica contra exploratoria - eran dramáticamente distintas. No nos sorprende que en la primera escuela no hubiera ningún efecto importante de la tecnología; en la segunda sí que se notó. De hecho, Harel (1988) demostró un contraste de este tipo entre alumnos que estudiaban Logo y quebrados en clase de matemáticas, de manera más didáctica en un grupo y de forma constructivista e integrada en el otro. Encontró que el segundo grupo logró un mayor dominio del Logo y una comprensión de quebrados mucho más profunda. O sea, no fue el Logo en sí lo que afectó las cogniciones, sino el Logo en un entorno cultural, orquestando los objetivos, integrándose con el tema de estudio y con los factores relacionados.

\section{CONCLUSIONES}

En esta época en que se construyen máquinas cada vez más inteligentes, nuestra pregunta inicial era si las máquinas puden hacer más inteligentes a las personas. La respuesta que proponemos es afirmativa, y en varios sentidos. Los efectos producidos CON la tecnología pueden re-definir y mejorar el rendimiento cuando los estudiantes trabajan en colaboración con las tecnologías inteligentes, esto es, aquellas que asumen una parte importante del proceso cognitivo que de otra manera correría a cargo de la persona. Además, los efectos $\mathrm{DE}$ la tecnología pueden producirse cuando la colaboración CON la técnica deja un residuo cognitivo, dotando a las personas de habilidades, y de estrategias del pensamiento que reorganizan y aumentan su rendimiento, incluso cuando estén apartadas de la tecnología en cuestión.

Esta respuesta afirmativa lleva, sin embargo, una advertencia importante: no es probable que se obtengan tales beneficios de forma automática con el avance de las tecnologías, más bien han de ser cultivados mediante el diseño apropiado de las tecnologías y de sus entornos culturales. Por ejemplo, aquellas tecnologías que ayudan a los usuarios a reorganizar sus cogniciones literarias para crear modelos nuevos y más eficaces (Zellermayer et al, 1990) pueden ayudar más que otras que no hacen más que proporcionar un ambiente cómodo de trabajo (por ej., un procesador de textos). Las tecnologías y los entornos culturales que 
fomentan la atención consciente tienen más posibilidades de producir un residuo cognitivo que aquellas que permiten al usuario caer en la distracción. Por otra parte, cuando hay beneficios, vienen acompañados de problemas que exigen soluciones - por ejemplo, los riesgos de una anulación de habilidades, la necesidad de una reconstrucción del concepto de inteligencia.

Naturalmente no debemos desanimarnos ante la necesidad de idear y proyectar para los beneficios, ni asustarnos por los enigmas que han de ser resueltos. Resulta demasiado ingenuo hablar de los efectos de la tecnología como un fenómeno natural e inevitable que ha de ser estudiado, registrado y analizado. Dentro de los límites de lo posible y lo práctico, los efectos de la tecnología. son los que elegimos para ella, y la responsabilidad de la decisión viene con la posibilidad de esta elección.

Por lo tanto, para aprovechar al máximo la oportunidad hace falta la colaboración, no sólo entre las personas y las máquinas, sino entre personas expertas en distintos campos. Al mismo tiempo que los investigadores en el campo de la inteligencia artificial continúan desarrollando instrumentos cognitivos, los expertos en la enseñanza, los investigadores de la pedagogía y el comportamiento humano, los sociólogos sensibles a los modelos de interacción cultural, e incluso los filósofos examinando cuestiones de significado y de ética, tendrán que dedicar sus opinones y sus conocimientos al problema. Pronosticamos un camino bastante tortuoso pero que llevará a resultados no sólo saludables sino también sorprendentes.

\section{Notas}

Una versión breve y modificada de este artículo fue presentada por Gavriel Salomon como conferenciante invitado en la reunión anual de la American Educational Researcb Association (abril, 1988) y parte se publicó en Proceedings of the 24th International Congress of Psychology (vol. 4). La redacción de este artículo fue financiada por una subvención de la Fundación Spencer concedida conjuntamente a Gavriel Salomon y la difunta Tamar Globerson, que murió de forma repentina y prematura mientras se estaba escribiendo este artículo.

\section{Referencias}

Anderson, J. R. (1983). The architecture of cognition. Cambridge, MA: Harvard University Press. Bereiter, C. y Scardamalia, M. (1987). The psychology of written composition. Hilldale, NJ: Erlbaum.

Bereiter, C., y TinKer, G. (1988, April). Consistency of constructive learning efforts. Symposium paper presented at the annual meeting of the AERA, New Orelans.

Bolter, D. J. (1984). Turing's man: Western culture in the computer age. Chapel Hill, NC: University of North Carolina Press.

Britton, B. K., Glynn, S. M., Meyer, J. F., y Penland, M. J. (1982). Effects of text structure on use of cognitive capacity during reading. Joumal of Educational Psychology, 74, 51-61.

Brown, J. S. Coluins, A., y Duguid, P. (1989). Situated cognitions and the culture of learning. Educational Researcher, 18, 32-42.

Cacioppo, J. T., y Petry, R. E. (1982). The need for cognition. Joumal of Personality and Social Psychology, 42, 116-131.

Chanowithz, B., Langer, E. J. (1980). Knowing more (or less) than you can show: Understanding control through the mindlessness-mindfulness distinction. In J. Garber y M.E.P. Seligman (Eds.), Human belplessness: Theory and application (pp. 97-129). Nueva York: Academic Press.

CH, M. T. H., Feltovich, P.J., y GASER, R. (1981). Categorization and representation of physics problems by experts and novices. Cognitive Science, 5, 121-152. 
Clements, D. H., y Gullo, D. F. (1984) Effects of computer programming on young children's cognitions. Joumal of Educational Psychology, 76, 1051-1058.

Cole, M. (1985). The zone of proximal development: Where culture and cognitions create each other. In J.V. Wertsch (Ed.), Culture, communication and cognition: Vygotskian perspectives (pp. 146-161). Nueva York: Cambridge University Press.

Cole, M., y Griffin, P. (1980). Cultural amplifiers reconsidered. En D.R. Olson (Ed.), The social foundations of language and thought: Essays in honor of J. S. Bruner (pp. 343-363). Nueva York: Norton.

DinUTE, C. (1985). Writing and computers. Reading, MA: Addison-Wesley.

Dinute, C. (1988, Mayo). Physical and cognitive factors in revision: Insights from studying with computers. Research in the Teaching of English, 22, 141-159.

Diaute, C. Y KRUIDENIER, J. (1985). A self-questioning strategy to increase young writer's revising processes. Applied Psycholinguistics, 6, 308-318.

ELLuL, J. (1964). The technological society. Nueva York. Knofp.

Gelman, R., y Brown, A. L. (1986). Changing competence in the young. In N. J. Smelser y D. R. Gesrstein (Eds.), Behavioral and social science: Fifty years of discovery (pp. 175-209). Washington, DC: National Academy Press.

GenTNER, D., y GENTNER, D. R. (1983). Flowing waters or teeming crowds: Mental models of electricity. In D. Gentner y A. L. Stevens (Eds.), Mental models (pp. 99-130). Hillsdale, NJ: Erlbaum.

Goody, J. (1977). The domestication of the savage mind. Nueva York: Cambridge University Press.

HAREL, I. (1988). Media design for leaning: Children's construction of meaning for fractions and Logo programming. Unpublished doctoral dissertation. Cambridge, MA: MIT.

Havelock, E. A. (1982). The literate revolution in Greece and its cultural consequences. Princeton, NJ: Princeton University Press.

Kozma, R. B., y VAN Roekel, J. (1986). Learging tool. Ann Arbor, MI: Arboworks.

Krendi, K. A., y Lieberman, D. A. (1988). Computers and learning: A review of recent research. Jorunal of Educational Computing Research, 4, 367-389.

LANGer, E. J. (1989). Mindfulness. Reading, MA: Addison-Wesley.

LEONTEV, A. N. (1981). The problem of activity in psychology. In J. V. Wertsh (Ed.), The concept of activity in Soviet psychology (pp. 37-71). Armonk, NY: Sharpe.

Levin, J. A., Ruel, M. M., MiYaKe, N., y CoHEN, M. (1987). Education on the electronic frontier: Teleapprentices in globally distributed educational contexts. Contemporary Educational Psychology, 12, 254-260.

MALONE, T. W., Y LEPPER, M (1987). Making learning fun: A taxonomy of intrinsic motivations for learning. In R. E. Snow y M. J. Farr (Eds.), Aptitude, learning and instruction: III. Conative and affective process analyses (pp. 223-253). Hillsdale, NJ: Erlbaum.

Mandinach, E. B. (1989). Model-building and the use of computer simulation of dynamic systems. Joumal of Educational Computing Research, 5, 221-243.

Mandinach, E. B. y CoRno, L. (1985). Cognitive engagement variations among students of different ability level and sex in a computer problem solving game. Sex Roles, 241-251.

MARCH, J. G. (1987). Old colleges, new technology. In S. B. Kiesler y L.Sl Sproul (Eds.), Computing and change on campus (pp. 16-27). Nueva York: Cambridge University Press.

MINTZ, R. (1988). Computer simulation as an instructional tool for the teaching of ecological systems. Unpublished doctoral dissertation. Tel Aviv University.

OLson, D. R. (1986). Intelligence and literacy: The relationship between intelligence and the technologies of representation and communication. In R. J. Sternberg y R. K. Wagner (Eds.), Practical intelligence: Nature and origins of competence in the everyday world (pp. 338-360). Nueva York: Cambridge University Press.

OLsun, D. R. (1988, Mayo). Mind and the techology of communication. Paper presented at the Australian Educational Conference.

Olson, D. R., Torrance, N., y HildyArd, A. (Eds.). (1985). Literacy, language and leaming. Cambridge, England: Cambridge University Press.

PAPERT, S. (1980). Mindstorms: Children, computers and powerful ideas. Nueva York: Basic Books.

PAPERT, S. (1987).Computer criticism vs. technocentric thinking. Educational Researcher, 17(1), 22-30.

PEA, R. D. (1985). Beyond amplification: Using the computer to reorganize mental functioning. Educational Psychologist, 20, 167-182.

PEA, R. D. (1987). Integrating human and computer intelligence. In R. D. Pea y K. Sheingold (Eds.), Mirrors of mind: Pattems of experience in educational computing (pp. 1?4.146! Norwood, NJ: Ablex.

PEA, R. D. (1989, June). Distributed intelligence and education. Documento presentado en el congreso anual de Social Science Research Council on Computers and Learning, British Virgin Islands.

PeA, R. D., KurLand, M. D. y Hawkins, J. (1985). LOGO and the development of thinking skills. En M. Chen y W. Paisley (Eds.), Children and microcomputers (pp. 193-212). Beverly Hills, CA: Sage.

Perkins, D. N. (1985). The fingertip effect: How information processing technology shapes thinking. Educational Researcher, 14(7), 11-17.

Perkins, D. N. y Salomon, G. (1987). Transfer and teaching thinking. In D. N. Perkins, J. Lock- 
head, y J. Bishop (Eds.), Thinking: The second international conference (pp. 285-303). Hillsdale: NJ: Erlbaum.

Perkins, D. N. y Salomon, G. (1989). Are cognitive skills context-bound? Educational Researcher, $18(1), 16-25$.

Richimond, B. (1985). STELLA. Lyme, NH: High Performance Systems.

Salomon, G. y Globerson, T. (1987). Skill may not be enough: The role of mindfulness in learning and transfer. International Joumal of Educational Research, 11, 623-638.

Salomon, G., Globerson, T., y Guterman, E. (1990). The computer as a zone of proximal development: Internalizing reading-related metacognitions from a Reading Partner. Joumal of Educational Psychology, 81, 620-627.

Salomon, G., y Leigh, T. (1984). Predispositions about learning from print and televison. Joumal of Communication, 20, 119-135.

Salomon, G. y Perkins, D. N. (1988). Tranfer of cognitive skills from programming: When and how. Joumal of Educational Computing Research, 3, 149-170.

Salomon, G., y Perkins, D. N. (1989). Rocky roads to transfer: Rethinking mechanisms of a neglected phenomenon. Educational Psychologist, 24, 113-142.

Sarason, S. B. (1984). If it can be studied or developed, should it be? American Psychologist, 39, 447.485.

SCARR, S. (1985). Constructing psychology: Making facts and fables of our times. American Psychologist, 40, 449-512.

SCHNEIDER, W., y FISK, D. (1984). Automatica category search and its transfer. Joumal of Experimental Psychology: Leaming, Memory, and Cognition, 10, 1-15.

SCrubner, S. y Cole, M. (1981). The psychology of literacy. Cambridge, MA: Harvard University Press.

SHIFFrin, R. M., y SCHNeIDER, W. (1977). Controlled and automatic human information processing: II. Perceptual learning, automatic attending, and a general theory. Psycbological Review, 84, $127-190$.

Simon, H. A. (1987). Computers and society. In S. B. Kiesler y L. S. y Sproul (Eds.). Computing and change on campus (pp. 4-15). Nueva York: Cambridge University Press.

VyGursky, L. S. (1978). Mind in society: The development of higher psychological processes. Cambridge, MA: Harvard University Press.

Zellermarer, M., Salomon, G., Globerson, R., y Givon, H. (en prensa). Enhancing related metacognitions through a computerized Writing-Partner. American Educational Research joumal.

\section{Coparticipando en el conocimiento:}

la ampliación de' la inteligencia humana

con las tecnologías inteligentes.

G. Salomon, D. N. Perkins y T. Globerson.

CL\&E, 1992, 13,pp. 6-22

Datos sobre los autores: Gavriel Salomon es profesor de Educación en la Universidad de $\Lambda$ rizona, especializado en educación y psicología cognitiva. David N. Perkins es co-director del Project Zero \& Senior Research Associate at Harvard Graduate School of Education. Tamar Globerson es profesor de educación en la escuela de educación de Tel-Aviv.

Dirección: Gabriel Salomon: University of Arizona, College of Education, Tucson, Arizona 85721. David N. Perkins: Harvard University, Project Zero, 315 Longfellow Hall, Cambridge, Massachusets 02138. Tamar Globerson: Tel-Aviv University. Tel-Aviv. Israel.

Articulo original: Partners in Cognition: Extending Human Intelligence with intelligent techonologies. Educational Researcher, Vol. 20, N. ${ }^{\circ}$ 3, Abril 1991, pp. 2-9. Reproducido con autorización. Traducción de Lucía Jones.

(C) De todos los artículos deberá solicitarse por escrito autorización de CL\&E y de los autores para el uso en forma de facsímil, fotocopia o cualquier otro medio de reproducción impresa. CL\&E se reserva el derecho de interponer las acciones legales necesarias en aquellos casos en que se contravenga la ley de derechos de autor. 